\title{
Modding de equipos informáticos como herramienta de aprendizaje en áreas STEM
}

\author{
Alberto Rodríguez Ruiz ${ }^{a}$, Nuria Ortigosa ${ }^{\text {b,c }}$ \\ ${ }^{a}$ Intituto de Enseñanza Secundaria Serpis, Valencia (Spain). rodriguez albrui@gva.es \\ ${ }^{\mathrm{b}}$ Departament d'Informàtica, Universitat de València (Spain). nuria.ortigosa@uv.es \\ ${ }^{\mathrm{c}}$ I.U. Matemática Pura y Aplicada, Universitat Politècnica de València (Spain). \\ nuorar@upvnet.upv.es
}

\section{Resumen}

El empleo de actividades que permitan mantener activo el interés al alumnado en distintas materias es un instrumento fundamental para mejorar el aprendizaje y ampliar conocimientos en todas las áreas educativas.

Modding, derivado de la jerga inglesa modify (modificar), es una técnica consistente en cambiar varios aspectos de equipos informáticos. Por un lado, hacer más atractiva la parte física del ordenador y, por otro, mejorar las prestaciones de dichos equipos. El objetivo a conseguir por parte de los estudiantes es mejorar, tanto visualmente como en términos de rendimiento, equipos informáticos, muchos de ellos en desuso, pero todavía funcionales. Para ello, mediante la presente actividad multidisciplinar de las áreas denominadas STEM (Science, Technology, Engineering and Mathematics), se desarrollan parte de los contenidos recogidos dentro del currículo docente.

Además, dicha interdisciplinariedad se amplía también a niveles de educación superior, haciendo partícipe del concurso al Departamento de Informática de la Universitat de València. En concreto, este último será el encargado de presentar a los participantes las distintas mejoras a nivel hardware y software que se pueden incluir para mejorar los resultados en términos de rendimiento de equipos, como por ejemplo la refrigeración líquida, o la importancia de la ventilación en sus prototipos.

Palabras clave: STEM, modding, herramienta aprendizaje, interdisciplinariedad. 


\section{Introducción}

La educación en áreas STEM necesita de instrumentos que permitan introducir nuevos conceptos de forma atractiva y efectiva en un alumnado necesitado de nuevas experiencias que le puedan acercar a sus intereses conocimientos específicos.

La propuesta de un concurso Modding nace de la idea de unir dichas necesidades de forma que el alumno se sienta partícipe del desarrollo de propuestas interesantes que, junto a la ayuda del profesor, le permitan adquirir nuevos conocimientos. Dicho concurso no deja de ser una tarea grupal entre alumnos del centro de distinto nivel y conocimiento, con el objetivo de aumentar el alcance de la actividad a toda la comunidad escolar.

El concurso se planteó a principios del curso como una actividad trimestral en la cual se habilitarían horarios y aulas dentro del centro para que el alumno pudiera acceder al material, involucrando a los departamentos de Matemáticas, Informática, Dibujo y Tecnología, los cuales cedían alternativamente y semanalmente parte de su horario a la realización de la actividad.

En este trabajo se presentan los contenidos desarrollados durante la actividad, siendo utilizado el concurso como herramienta motivadora, trabajando en grupos de 3 a 5 alumnos, con premios para todos los participantes.

\section{La tecnología Modding en educación}

\subsection{Modding como instrumento de modficicación de equipos informáticos}

Modding, derivado de la jerga inglesa modify, es una combinación de distintas técnicas cuyo objetivo principal es personalizar el ordenador personal (PC) de forma que se mejore tanto estética como funcionalmente las prestaciones del equipo informático.

Dichas técnicas suelen abarcar modificaciones de tipo hardware y software, dotando al equipo de unas características que lo hacen diferente de todos los demás.

Desde el punto de vista estético, el arte modding consiste en hacer más atractivo el equipo según los gustos del modder o aficionado al modding. Dichas modificaciones incluyen el cambio de la carcasa de la torre del PC, inclusión de iluminación LED, o destacar una utilidad adicional a la del propio $\mathrm{PC}$, junto con los periféricos que le pueden acompañar. 
Ateniéndonos también a la modificación interna, esta técnica también abarca las modificaciones que mejoren el rendimiento del equipo, siendo muy usual la inclusión de refrigeración líquida para mejorar el rendimiento.

\subsection{Estrategias de aprendizaje a través de modding informático}

La actividad consiste principalmente en un concurso abierto a todo el alumnado dentro del mismo centro de forma grupal con el objetivo de fomentar las interacciones entre alumnos de un mismo o distinto nivel. La actividad es constructivista, donde el propio alumno es el que proporciona las herramientas y equipos a modificar. La libertad de la tarea abre un gran abanico de posibilidades en la creatividad e imaginación de todos ellos.

Las actuaciones del profesorado durante la actividad consistirán principalmente en guiar el proceso dentro de los cauces normalizados establecidos, pudiendo corregir cualquier desviación dentro del planteamiento inicial. La inclusión de mejoras técnicas hardware harán partícipe al Departamento de Informática de la Universitat de València, encargado de presentar a los participantes las distintas mejoras a nivel hardware y software que se pueden incluir para mejorar el rendimiento de equipos.

\section{Enfoque educativo de la actividad para las áreas STEM}

\subsection{La actividad dentro del currículo de secundaria.}

Tanto el manejo, como modificación de equipos informáticos se encuentran presentes y se tratarán en la actividad, quedando presentes especialmente los contenidos del Decreto $87 / 2015$, de 5 de junio por el que se establece el currículo de educación secundaria, cuyo bloque de contenidos que trabajará la actividad serán los siguientes:

- Describir los tipos de equipos informáticos y sus componentes básicos en un entorno cotidiano y clasificarlos según su funcionalidad.

- Analizar la arquitectura de un equipo informático, identificando los componentes físicos y periféricos y describiendo sus características y procedimientos de conexión para su aplicación en un entorno cotidiano.

- Reconocer la terminología conceptual de la asignatura y del nivel educativo y utilizarla correctamente en actividades orales y escritas del ámbito personal, académico, social o profesional. 
- Argumentar los cambios tecnológicos más relevantes para valorar su repercusión tanto tecnológica como económica y social, en base a documentación escrita y digital.

- Participar en intercambios comunicativos del ámbito personal, académico (resolución de problemas en grupo), social o profesional aplicando las estrategias lingüísticas y no lingüísticas del nivel educativo propias de la interacción oral, utilizando un lenguaje no discriminatorio.

- Identificar los valores medioambientales y estéticos como parte de la diversidad cultural y respetarlos contribuyendo a su conservación y mejora.

\section{Análisis de resultados de la experiencia}

\subsection{Resultados del concurso}

Cada equipo presentó la unidad de forma que fuera funcional junto con una memoria explicativa de todo el desarrollo. El último día de concurso se realizó una exposición de los trabajos por parte de cada grupo, siendo el público y jurado, tanto el propio alumnado como una representación de los profesores de áreas STEM que han sido partícipes, una representación del AMPA, así como de una empresa colaboradora que patrocinó el evento y premió a los participantes según la categoría.

Durante la presentación se pudieron destacar las funcionalidades de los equipos así como las principales características que los hacían particulares a cada uno.

Tras deliberación y posterior votación del jurado, se obtuvieron los siguientes resultados entre los 5 finalistas, quedando como equipo ganador el proyecto mostrado en la Figura 1: 
Tabla 1. Resultados votación del concurso. Fuente: votación del jurado.

\begin{tabular}{|c|c|c|c|c|c|c|}
\hline \multicolumn{2}{|c|}{$\begin{array}{l}\text { Valoración del jurado } \\
\text { (sobre } 8 \text { puntos) }\end{array}$} & $\begin{array}{c}\text { GRUPO } \\
1 \\
\end{array}$ & $\begin{array}{c}\text { GRUPO } \\
2 \\
\end{array}$ & $\begin{array}{c}\text { GRUPO } \\
3 \\
\end{array}$ & $\begin{array}{c}\text { GRUPO } \\
4\end{array}$ & $\begin{array}{c}\text { GRUPO } \\
5\end{array}$ \\
\hline \multicolumn{2}{|c|}{ Aspectos a valorar } & $(0-10)$ & $(0-10)$ & $(0-10)$ & $(0-10)$ & $(0-10)$ \\
\hline \multirow{5}{*}{$\begin{array}{c}\text { Equipo } \\
\text { (sobre } 5 \\
\text { ptos.) }\end{array}$} & Originalidad & 9.3 & 7.9 & 7.4 & 6.6 & 6.7 \\
\hline & Aspecto visual & 9.7 & 7.7 & 7 & 7 & 6.1 \\
\hline & $\begin{array}{c}\text { Materiales } \\
\text { usados }\end{array}$ & 9.1 & 8.7 & 7.3 & 7 & 6 \\
\hline & Funcionalidad & 7.4 & 9.8 & 7 & 7.4 & 6.4 \\
\hline & $\begin{array}{l}\text { Acceso a los } \\
\text { componentes }\end{array}$ & 7.9 & 8.7 & 5.7 & 6.5 & 7.85 \\
\hline \multirow{4}{*}{$\begin{array}{c}\text { Memoria } \\
\text { (sobre } 2 \\
\text { ptos.) }\end{array}$} & Contenidos & 10 & 9.8 & 6.8 & 6.6 & 9 \\
\hline & $\begin{array}{l}\text { Presentación y } \\
\text { maquetación }\end{array}$ & 9.2 & 9.8 & 6 & 6.6 & 8.2 \\
\hline & $\begin{array}{c}\text { Claridad } \\
\text { descriptiva }\end{array}$ & 9.6 & 10 & 6.2 & 6.8 & 7.4 \\
\hline & $\begin{array}{l}\text { Detalle de } \\
\text { planos }\end{array}$ & 8.2 & 9 & 4.4 & 6 & 8.2 \\
\hline \multirow{2}{*}{$\begin{array}{l}\text { Presentación } \\
\text { (sobre } 1 \text { pto.) }\end{array}$} & $\begin{array}{c}\text { Calidad del } \\
\text { montaje }\end{array}$ & 8.4 & 9.8 & 6.6 & 6.8 & 7.4 \\
\hline & Exposición & 9.2 & 10 & 6.2 & 7.6 & 7.6 \\
\hline \multicolumn{2}{|c|}{ TOTAL JURADO } & 7.07 & 7.2 & 5.25 & 5.47 & 5.695 \\
\hline \multicolumn{2}{|c|}{$\begin{array}{l}\text { Votación popular } \\
\text { (sobre } 2 \text { puntos) }\end{array}$} & 2 & 0.91 & 0.5 & 0.24 & 0.03 \\
\hline \multicolumn{2}{|c|}{ TOTAL } & 9.07 & 8.11 & 5.75 & 5.71 & 5.725 \\
\hline \multicolumn{2}{|c|}{ Posición } & 1 & 2 & 3 & 5 & 4 \\
\hline
\end{tabular}

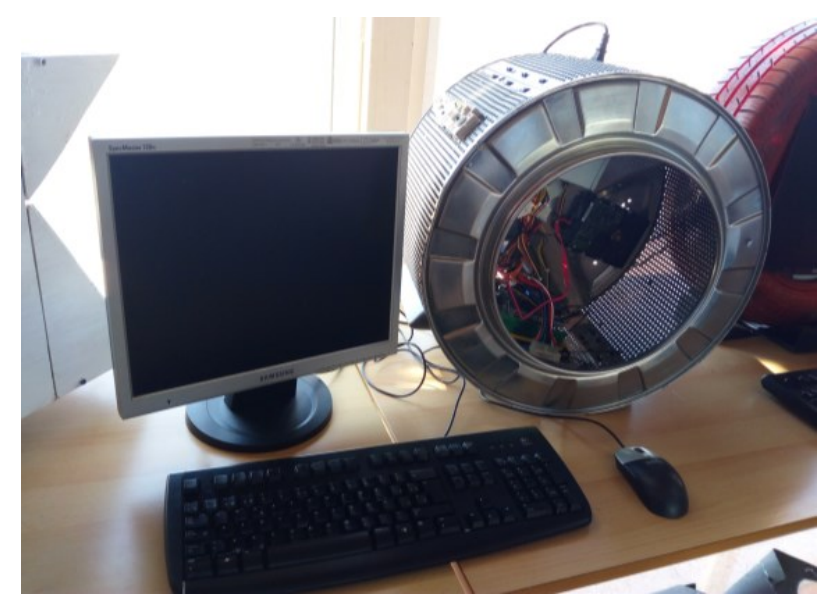

Fig. 1 Proyecto ganador del concurso. Fuente: elaboración propia. 


\section{Conclusiones y trabajo futuro}

En este artículo hemos presentado los conceptos y la estructura del desarrollo de la actividad/concurso modding que se ha realizado para que alumnos de enseñanzas medias tengan contacto con las actividades de innovación y creación dentro de áreas STEM.

La actividad consiguió el objetivo planteado inicialmente, obteniendo una gran aceptación y participación de todos los miembros de la comunidad educativa.

Esta actividad, novedosa dentro de la educación secundaria, ha obtenido buenos resultados, reflejados por una parte en la alta participación de todo el alumnado de centro y en su aplicación de los conceptos estudiados previamente.

Además, tras finalizar la actividad, los alumnos expusieron el trabajo que han desarrollado y los conceptos nuevos que han aprendido durante el desarrollo de la misma. Como limitaciones a la misma, se plantea el acceso a los diferentes materiales por parte de los alumnos para su empleo en la actividad, así como la dedicación horaria adicional para poder terminar en plazo los trabajos.

Queda como trabajo futuro, integrar nuevas áreas y departamentos a la actividad, así como una propuesta intercomarcal entre distintos centros educativos próximos para compartir la experiencia y unificar el concurso en uno solo.

\section{Referencias}

Aguilera López, Purificación. (2010). Seguridad informática. Editorial Editex.

Aspinwall, Jim. (2004). PC hacks. O'Reilly Media, Inc.

El-Nasr, Magy Seif; Smith, Brian K. (2006). Learning through game modding. Computers in Entertainment, 4.

Groth, David (2004). PC Chop Shop: Tricked Out Guide to PC Modding. Sybex Inc .

Hardwidge, Ben (2006). Building extreme PCs : the complete guide to computer modding. O'Reilly Media, Inc. 